Lexis Vol. XLIV (2) 2020: 659-698

\title{
Las construcciones negativas en mapudungun. Una mirada tipológica*
}

\author{
Aldo Olate \\ Universidad de La Frontera \\ Fernando Zúñiga \\ Universität Bern \\ Rodrigo Becerra \\ Universidad de Alberta
}

\section{RESUMEN}

Este trabajo explora las construcciones negativas del mapudungun desde la lingüística tipológica contemporánea. A partir de datos recogidos en el campo y de una discusión bibliográfica previa, se establecen los esquemas de la negación prototípicos del mapudungun y sus variaciones. Finalmente, se propone una clasificación tipológica de las construcciones de la lengua de acuerdo con la negación estándar y no estándar, los indefinidos negativos, el tipo de mecanismo que utiliza la lengua. Se concluye con algunas

\footnotetext{
"El presente trabajo se realizó gracias al apoyo del proyecto FONDECYT 1180071: "TAM, evidencialidad, fenómenos asociados a la valencia verbal y la codificación de eventos complejos en el castellano de contacto hablado por bilingües mapuche/castellano. Un estudio comparativo del sistema verbal”.
}

https://doi.org/10.18800/lexis.202002.008 
reflexiones generales sobre el fenómeno de la negación en mapudungun y sus dimensiones tipológicas y areales.

Palabras clave: construcciones negativas, negación estándar, tipología lingüística, mapudungun

Negative Constructions in Mapudungun: A Typological Approach

\section{ABSTRACT}

In this paper, we explore the negative constructions in Mapudungun from the perspective of current linguistic typology. Based on published sources and fieldwork data, we establish the prototypical negative schemes and their variation in Mapudungun. We propose a typological classification of the constructions in agreement with the standard negation, negatives pronouns, and the types of mechanism that the language has for expressing the negation. Finally, surveyed and advance some general reflections on this phenomenon in the language.

Keywords: negative constructions, standard negation, linguistic typology, Mapudungun

\section{Presentación}

La negación es una categoría presente en todas las lenguas del mundo, pero cada sistema tiene sus propias estrategias para codificarla (Dahl 2011). Aun cuando diversas gramáticas han descrito los rasgos de la negación en mapudungun, no se ha llevado a cabo el ejercicio comparativo o tipológico que nos permita establecer las particularidades de este dominio en la lengua mapuche. Los motivos de esta ausencia tienen relación, principalmente, con el desarrollo reciente de los estudios tipológicos en lo relacionado con la negación.

La descripción de las construcciones negativas del mapudungun hablado en el área de la Araucanía (Chile) que presentamos en este trabajo es relevante para enriquecer nuestro conocimiento de las lenguas indoamericanas y surandinas, además del conocimiento tipológico en general. En primer lugar, permite situar la lengua 
mapuche en el marco de la tipología de las estructuras negativas; en segundo lugar, pone a disposición insumos para establecer posibles relaciones lingüísticas areales y, finalmente, provee datos que enriquecen el conocimiento de las propias tipologías propuestas. En este marco, este estudio da cuenta de algunas estructuras negativas vigentes en la lengua mapuche; más específicamente, se analizan estrategias de negación estándar y no estándar en construcciones finitas, no finitas y no verbales. En el marco de las primeras, se presta atención a las formas de negación predicativas y estativas, con la finalidad de centrarse en las indicativas, subjuntivas e imperativas. En las segundas, se revisan las atributivas, locativas, inclusivas propias, existencial y posesivas. Finalmente, se da cuenta del comportamiento de los indefinidos negativos.

Esta exposición se organiza de acuerdo a las siguientes secciones: (2) la tipología de la negación en general; (3) revisión de estudios sobre construcciones negativas en el mapudungun; (4) la investigación particular, donde se presentan la muestra y los criterios de análisis; y (5) la descripción lingüística de los esquemas que se encuentran en nuestro corpus.

\section{Aspectos generales de la negación}

En términos generales (y simples), la negación es un operador que cambia el valor de verdad de una proposición. Sin embargo, este fenómeno exhibe complejas interacciones con diversos aspectos estructurales y semánticos; vista desde una perspectiva tipológica, la negación es un fenómeno complejo aplicado sobre dominios morfosintácticos diversos (Miestamo 2005, 2007). No pretendemos aquí profundizar en los aspectos filosóficos del fenómeno, sino más bien comentar brevemente las tipologías de la negación propuestas en la literatura ${ }^{1} \mathrm{y}$, a partir de allí, ver a cuáles patrones se ajusta el mapudungun.

\footnotetext{
Los estudios sobre la negación desde una perspectiva tipológica son numerosos. Véase Miestamo $(2005,2007,2017)$ y Dahl (2011) para una revisión bibliográfica detallada.
} 
El ámbito de la negación y su actualización estructural es amplio; por ello, para ingresar a este campo, se debe observar cómo esta actúa sobre las distintas construcciones de las lenguas (p. e. predicativas, estativas, no finitas, no verbales, léxicas) y sus propiedades gramaticales (p. e. TAM). También, debemos considerar el tipo de elemento (morfema o partícula) que opera como negador en el nivel de la cláusula. Por otro lado, hay que tener en cuenta el funcionamiento de los pronombres negativos indefinidos (p. e. 'nadie', 'nada', 'ninguno', 'nunca').

A nivel clausal, la negación puede presentar formas diversas según el modo y la finitud del predicado verbal, así como según si el predicado (y la cláusula) es verbal o no. Hay que agregar que la lengua puede recurrir a estrategias sintácticas o morfológicas para marcar el fenómeno. También, es relevante la posición que ocupa la marca de negación en la cláusula. Hay lenguas en que la partícula negativa aparece antes del verbo o después de él. Lo mismo ocurre a nivel morfológico, donde la negación puede ser prefijal, sufijal o circunfijal, dependiendo de la lengua ${ }^{2}$ (Dryer 2013a). Otros dos aspectos que hay que tener presentes se relacionan con el funcionamiento de los pronombres negativos indefinidos y los procesos de derivación léxica.

En consecuencia, desde la perspectiva tipológica, la negación es un fenómeno mucho más complejo que la idea de aplicar un mero marcador de negación sobre una oración declarativa. Antes de ingresar al detalle descriptivo de la lengua, creemos necesario dar cuenta de las miradas que hay sobre la negación desde la tipología. El repaso se inicia con la idea de negación estándar desarrollada por Miestamo (2005, 2007, 2013a, 2013b, 2017). Luego abordamos la clasificación propuesta por Dryer (2013a, 2013b, 2013c), y la de Haspelmath (2013) de los indefinidos negativos (enmarcadas en el

\footnotetext{
2 En este trabajo no consideramos el campo de la negación afijal entendida como parte de procesos de formación de palabras y que involucra elementos tales como des- (p. e. en descontento) in- (p. e. en inmaterial), que se adscriben al plano léxico derivativo (Dahl 2011).
} 
proyecto WALS del Instituto Max Planck). Esta sección finaliza con la negación no estándar.

\subsection{La negación estándar}

La falta de homogeneidad en las formas de negación es uno de los problemas más complejos en este dominio tipológico, lo que ha llevado a la propuesta de diversas etiquetas para la tipología. El punto de partida ha sido observar las construcciones negativas básicas en los distintos tipos de lenguas (Dahl 2011). En este marco, surge la noción de negación estándar, introducida por Payne (1985), que se refiere al modo en que opera la negación en las cláusulas de indicativo. Las formas que adopta en los otros modos y tipos de construcciones reciben el nombre de negación no estándar (Miestamo 2005, 2007, 2013a, 2013b, 2017).

La negación puede ser simétrica o asimétrica. En el caso de simetría estructural, la cláusula negativa de indicativo difiere estructuralmente de la afirmativa, puesto que añade un operador negativo (sea este un afijo o una palabra). Por el contrario, en el segundo caso, la distinción no depende solo de la presencia de un operador negativo, sino que se aprecian asimetrías estructurales entre las cláusulas de indicativo negativas y afirmativas. Así, se distinguen tres subtipos: asimetría que involucra la finitud del verbo, asimetría que implica la marca negativa como irrealis (no realizado), y asimetría que involucra un cambio en categorías gramaticales de TAM y de persona. Además del punto de vista estructural, centrado en construcciones específicas, el parámetro de simetría puede considerarse desde el punto de vista de los paradigmas en su conjunto. Los paradigmas simétricos implican la correspondencia de todas las formas negativas y afirmativas, sin importar su simetría o asimetría estructural. Los asimétricos, en cambio, no presentan dicha correspondencia uno a uno (Miestamo 2005, 2013a, 2013b).

Por ejemplo, en (1a-d) podemos observar un caso de negación estándar estructuralmente simétrica con paradigma también simétrico. La forma de la cláusula no distingue estructuralmente entre 
construcciones afirmativas y negativas. Además, se muestra simetría en el paradigma, pues todas las formas afirmativas pueden ser negadas (Miestamo 2013a):

(1) Español (indoeuropea, romance) (Ejemplo propio)
a. Rodrigo
baila
rancheras.
Rodrigo
bailar.3SG.IND.PRES
ranchera.PL ${ }^{3}$
b. Rodrigo
bail-ó
Rodrigo
bailar-3SG.IND.PST
rancheras.
c. Rodrigo
no bail-a
Rodrigo
NEG bailar-3SG.IND.PRES
ranchera.PL
d. Rodrigo
no bail-ó
rancheras.
ranchera.PL
Rodrigo
NEG bailar-3 SG.IND.PST
rancheras.
ranchera.PL

En cuanto a la negación estándar estructuralmente asimétrica, el finlandés presenta el siguiente esquema:

(2) Finlandés (urálica) (Miestamo 2013a)
venir-1SG
'Vengo.'
a. Tule-n
b. $E-n$
NEG-1SG
'No vengo.'
tule.
venir.CONNEG.PRES
c. Tul-i-n
venir-PST-1sG
'Vine.'
d. $E-n$
NEG-1SG
'No vine.'
tul-lut.
venir.PST.PTCP

En (2), existe un contraste estructural entre las formas afirmativas y las negativas. En las últimas, la negación es marcada por un

\footnotetext{
3 Las abreviaturas utilizadas en este trabajo son las siguientes: $1=$ primera persona; $2=$ segunda persona; 3 = tercera persona; $\mathrm{ABS}=$ absolutivo; $\mathrm{ACC}=$ acusativo ACT $=$ activo; $\mathrm{ADIT}$ $=$ aditivo; $\mathrm{APRF}=$ antiperfecto; $\mathrm{ASS}=$ asertivo $; \mathrm{AUX}=$ auxiliar , $\mathrm{CONNEG}=$ connegativo $\mathrm{COP}$ = cópula $; \mathrm{DEM}=$ demostrativo; $\mathrm{DET}=$ determinante; $\mathrm{DIR}=$ direccional $; \mathrm{ENF}=$ enfatizador; $\mathrm{EPI}=$ epistémico; $\mathrm{EST}=$ estativo; $\mathrm{EVID}=$ evidencial $; \mathrm{EX}=$ existencial; $\mathrm{FUT}=$ futuro; $\mathrm{HAB}=$ habitual; IMP = imperativo; INCL = inclusivo; INCOMPL = incompletivo; INF = infinitivo; INS $=$ instrumental; $\mathrm{INTR}=$ intransitivo; $\mathrm{IRR}=$ irrealis; IRR.PST $=$ irrealis pasado; $\mathrm{IND}=$ indicativo; LOC= locativo; $\mathrm{NEG}=$ polaridad negativa; $\mathrm{NFIN}=$ no finito; $\mathrm{NTR}=$ aspecto neutro; $\mathrm{PERF}=$ perfecto; $\mathrm{PST}=$ pasado; $\mathrm{PL}=$ plural; $\mathrm{POS}=$ posesivo $;$ POSP $=$ posposición $;$ PRES $=$ presente; PTCP = participio; R.PST.PUNCT $=$ realis pasado puntual $;$ R.PST.CONT $=$ realis pasado continuo; $\mathrm{S}$ = sujeto intransitivo; $\mathrm{SBJV}=$ subjuntivo; $\mathrm{SG}=$ singular; $\mathrm{TOP}=$ tópico.
} 
verbo auxiliar negativo $e^{-}$, el cual toma las marcas de persona y número, y el verbo léxico tulea 'venir' pierde su finitud, y aparece en la forma presente "con-negativa" (2b) y en participio de pasado (2d). Sin embargo, el paradigma de negación es simétrico, porque todas las afirmativas pueden ser negadas con el auxiliar negativo $e$ (Miestamo 2005, 2013a, 2007).

La negación de paradigmas asimétricos se observa en (3), donde el paradigma afirmativo distingue entre presente, futuro y perfecto pero dicha distinción se pierde en la construcción negativa, ya que en esta se reemplazan los sufijos que marcan las categorías temporoaspectuales por el circunfijo ma $b \hat{u}$.

(3) Birmano (tibeto-birmana) (Miestamo 2013a)
a. $\theta w a-d e ́$.

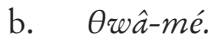
ir-PRES ir-FUT
'Va.'
'Irá.'
c. $\quad$ irâ-bí.
ir-PERF
'Ha ido.'
d. $M a-\theta w \hat{a}-b \hat{u}$. NEG-ir-NEG 'No va/irá/ha ido.'

La circunfijación por medio del morfema ma...bu implica el reemplazo de las marcas TAM en (3a) (dé), (3b) (mé) y (3c) (bi). Las marcas negativas no se añaden simplemente a la correspondiente forma afirmativa. El paradigma, por su parte, también es asimétrico, pues en el modelo afirmativo se hace una distinción aspectotemporal entre presente, futuro y perfecto, la que se pierde en la construcción negativa al añadir los circunfijos (Miestamo 2013a). Por otro lado, hay lenguas que tienen un tipo de negación mixto simétrico-asimétrico; es decir, la negación estándar es simétrica en algunas construcciones y asimétrica en otras (Miestamo 2013a).

Por último, la negación puede tener simetría estructural y asimetría paradigmática. Este caso lo vemos en (4), donde, por un lado, se observa $(4 \mathrm{c}-\mathrm{d})$ que solo se agrega la marca negativa marig sobre la forma irrealis afirmativa, lo que produce simetría estructural. 
(4) Maung (yiwaidjana) (Miestamo 2013a)
a. Ni-udba- $\eta$.
1sG.3-poner-R.PST.PUNCT
'Lo puse.'

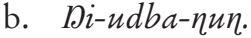
1SG.3-poner-R.PST.CONT
'Lo estaba poniendo.'
c. $\quad d a \quad \eta i-u d b a-n j i$
si 1sG.3-poner-IRR.PST
'si lo pusiera'
d. marig $\eta i-u d b a-n j i$
NEG 1SG.3-poner-IRR.PST
'si no lo pusiera'

Por otro lado, la asimetría paradigmática se observa entre el modo realis y el irrealis, pues la distinción continuo-puntual del primero $(4 a-b)$ se pierde en el irrealis $(4 c-d)$.

A continuación, un resumen de la clasificación basada en el parámetro de simetría se puede apreciar en la tabla 1 :

Tabla 1. Construcciones negativas estándar según su simetría

\begin{tabular}{lll}
\hline & Estructural & Paradigmática \\
\hline Castellano & Simétrica & Simétrica \\
Finlandés & Asimétrica & Simétrica \\
Birmano & Asimétrica & Asimétrica \\
Maung & Simétrica & Asimétrica \\
\hline
\end{tabular}

Miestamo (2007) afirma la utilidad de esta clasificación para conceptualizar las construcciones negativas, pues, a partir de ella, pueden explicarse funcionalmente los distintos tipos de negación estándar. El primer criterio para explicar las asimetrías es aspectual (estatividad vs. dinamicidad): las cláusulas afirmativas pueden expresar situaciones estativas o dinámicas, frente a las negativas, que prototípicamente dan cuenta de estativas. El segundo se refiere al estatus de realidad: la cláusula expresa lo realmente realizado, mientras que la negativa, lo no realizado. El tercero guarda relación con el contexto del discurso: la construcción negativa es utilizada en contextos donde la correspondiente afirmativa está presupuesta de algún modo. A partir de estos criterios y considerando procesos 
analógicos, se postulan las explicaciones de los tipos de asimetrías ${ }^{4}$ (Miestamo 2007: 559).

\subsection{Morfemas negativos}

Dryer (2013a) muestra los tipos de morfemas que se especializan en la negación. Hay cuatro grupos en esta tipología: (a) la negación indicada por medio de afijos; (b) la negación expresada a través de palabras autónomas, con cuatro subtipos (partículas negativas, auxiliares negativos, verbos negativos y palabras negativas de estatus incierto entre verbo y partícula); (c) la negación que varía entre afijo y palabra, y (d) la doble negación.

En el ejemplo (5), se observa una negación afijal; el marcador negativo es el circunfijo $n d(a)-\ldots-i^{5}$ para oraciones declarativas:

(5) Guaraní (tupí, tupí-guaraní) (Krivoshein de Canese 1983)
a. (Che) a-guata.
1sG 1sG.ACT-caminar
'(Yo) camino.'
b. (Che) nd-a-guata-i.
1sG NEG-1sG.ACT-caminar-NEG
'(Yo) no camino.'

La parte prefijada se manifiesta en el margen izquierdo del verbo, antes de los marcadores de persona, mientras que la parte sufijada es concatenada antes de los marcadores aspectuales y del sufijo intensificador - ete, en el caso de estar estos presentes (Estigarribia 2017: 56).

\footnotetext{
4 Para revisar con detalle las explicaciones funcionales, consúltese Miestamo (2007: 558-560).

5 Este morfema tiene alomorfos que dependen de la morfofonología de la raíz verbal y de los marcadores de persona. Por ejemplo, con raíces nasales se usa $n(a)-\ldots-i$, y la vocal de la parte prefijada suele experimentar armonía vocálica con los marcadores de persona (Estigarribia 2017). La negación de oraciones imperativas, optativas y subordinadas también es morfológica, realizada principalmente mediante circunfijos, aunque con ciertas excepciones. Por ejemplo, la parte prefijal de la negación usada en modo imperativo es a veces analizada como palabra separada del verbo (adverbio).
} 
La negación expresada por medio de palabras independientes tiene cuatro subtipos. En el ejemplo (1), del castellano, se observa el primero, a saber: el caso de las partículas negativas (la gramática tradicional del castellano habla de "adverbios negativos"). Otro caso similar se observa en kawésqar, donde la mayoría de las construcciones predicativas son negadas mediante la partícula k'élok que se posiciona después del verbo (6):

(6) Kawésqar (aislada) (Aguilera y Tonko 2006)
a. Fčakiáns sa
čo jeksór.
zorzal
TOP
1SG ver
'Yo veo un zorzal.'

$\begin{array}{lllll}\text { b. Fčakiáns } & \text { sa } & \text { čo } & \text { jéksor } & \text { k’élok. } \\ \text { zorzal } & \text { TOP } & \text { 1sG } & \text { ver } & \text { no }\end{array}$
'Yo no veo un zorzal.'

En (2), del finlandés, se aprecia el segundo subtipo, con un auxiliar negativo que aparece junto con un participio con-negativo. El tercer subtipo consiste en un verbo negativo que toma la flexión de tiempo del verbo de la cláusula afirmativa (7a-b).

(7) Grebo (niger-congo, kru) (Dryer 2013a)
a. Ne $d u-d a$
bla.
1sG.SUBJ
'No golpeé el arroz antes de ayer.'
b. Ne $y i-d a$
bla
$d u$
mole.
1sG.SuBJ
NEG-PST.ANTES.AYER
arroz
golpear lunes
'No golpeé el arroz el lunes.'

Finalmente, en el cuarto subtipo no hay claridad sobre si el negador es un verbo o una partícula $(8)^{6}$ :

\footnotetext{
6 El estatus del negador 'ína es incierto. De acuerdo a Kieviet (2017), se ha aducido que podría corresponder a un verbo que tiene por complemento una cláusula subordinada, tal como ocurre en otros idiomas polinésicos. Sin embargo, el autor propone que, tal como inau (un posible cognado en la lengua mangareva), 'ina podría haberse originado
} 
(8) Rapa nui (austronésica, oceánica) (Kieviet 2017)

a. He u'i rō mai a Heru

hai mata.

NTR mirar ENF hacia.acá DET Heru

INS ojo

'Heru (lo/la) miró con sus ojos.'

b. 'Ina a Heru be u'i rō mai

bai mata.

NEG DET Heru NTR mirar ENF hacia.acá

INS ojo

'Heru no (lo/la) miró con sus ojos.'

La clase de negación que cuenta con un afijo para un tipo de construcción y una partícula para otra se aprecia en (9a-b). La lengua presenta variación entre el afijo taama y la palabra negativa aa:

(9) Rama (chibcha, rama) (Dryer 2013a)

a. I-sik-taama.

3-llegar-NEG

'No llegó.'

b. Nkiikna-lut unt aa kain-i.

hombre-PL gallo NEG hacer-TNS

'El hombre no hizo el gallo.'

Finalmente, se encuentran los casos de doble negación que involucran dos morfemas simultáneos (10-12); frecuentemente, uno precede al verbo mientras que el otro lo sigue, aunque puede ocurrir que ambos estén en una misma posición respecto al verbo (Dryer 2013a).

(10) Francés (indoeuropea, romance) (Dryer 2013a)

$\begin{array}{lllll}\text { Je ne vois } & \text { pas la } & \text { lune. } \\ \text { 1SGNEG } & \text { veo } & \text { NEG la } & \text { luna }\end{array}$

'No veo la luna.'

como marcador independiente de polaridad y luego haber asumido las funciones de negador clausal. 
(11) Quechua (quechua) (Cerrón-Palomino 20087)

a. Chay runa-qa tiyu-y-mi.

DEM hombre-TOP tío-POS.1SG-EVID

'Ese hombre es mi tío.'

b. Chay

runa-qa mana-m

DEM hombre-TOP

NEG-EVID

tiyu-y-chu.

'Ese hombre no es mi tío.'

(12) Aymara (aymara) (Grondin 1999)

a. Aka-x chu'qi-wa.

DEM-TOP papa-EVID

'Esto es (una) papa.'

b. Aka-x jani-w

DEM-TOP NEG-EVID

'Esto no es (una) papa.'

chu'qi-k-i-ti.

papa-INCOMPL-3SG-NEG

En cuanto al orden de los morfemas negativos, este puede ser preverbal, postverbal, doble negación, doble o triple negación opcional (Dryer 2013b). Asimismo, la posición de las palabras negativas respecto de sujeto, verbo y objeto da cuenta de una amplia gama de posibilidades combinatorias, tomando como base los tipos de organización de constituyentes (SVO, SOV), y los morfemas y palabras especializados para la negación ${ }^{8}$ (Dryer 2013c).

\subsection{Los pronombres indefinidos negativos}

Los pronombres indefinidos negativos se entienden como expresiones nominales o adverbiales que se traducen directamente como 'nadie', 'nada' o 'nunca', independientemente de si ocurren simultáneamente con la negación predicativa (Haspelmath 2013).

\footnotetext{
7 Cabe consigar que Cerrón Palomino (2008) no utiliza una variante particular del quechua, sino una normalización de este a partir de diversas variantes, especialmente sureñas (quecua II-C) y, además, de datos históricos.

${ }_{8}$ No es el objetivo de este artículo profundizar en esta extensa clasificación. El lector puede consultar en el WALS el capítulo de Dryer sobre la posición de los morfemas negativos respecto al sujeto, objeto y verbo.
} 
En cuanto a los pronombres negativos indefinidos (p. e. nadie, nada), estos pueden o no ocurrir con las marcas generales de negación predicativa. Hay lenguas en que ambos elementos tienen que ocurrir simultáneamente y la omisión del negador predicativo puede generar agramaticalidad (Haspelmath 2013).

En (13a-b) se observa que tanto el pronombre negativo como la marca de negación predicativa pueden aparecer en la misma cláusula:

(13) Guaraní (tupí-guaraní) (Estigarribia 2017)
a. Ai-kuaa.
$\begin{array}{ll}\text { b. } \quad N d-a i-k u a a-i & m b a ' e v e . \\ \text { NEG-1sG.ACT-saber-NEG } & \text { nada }\end{array}$
1sG.ACT-saber
'Yo sé.'
'Yo no sé nada.'

De manera similar, en quechua y aymara también aparecen marcas de concordancia negativa en contextos de pronombres de interpretación indefinida. Sin embargo, a diferencia del guaraní (13), en estas lenguas el pronombre no es negativo, sino que la concordancia negativa se logra en (14-15) mediante la concurrencia de la partícula negativa más los sufijos de negación, tal como en los ejemplos (11-12):

(14) Quechua (quechua) (Taylor 2014')
a. Mana riku-ni-chu.
NEG ver-1SG-NEG
'No veo.'
b. Mana ima-kta-pas ${ }^{10}$
NEG qué-ACC-ADIT
'No veo nada.'

\author{
riku-ni-chu. \\ ver-1sG-NEG
}

\footnotetext{
9 Debe señalarse que Taylor (2014) reporta un quechua llamado "lengua general”, el cual es utilizado como lengua franca durante la segunda mitad del siglo XVI y la primera XVII.

10 El sufijo ( $k$ )ta, cuyo alomorfo - $k t a$ es usado después de vocal en el manuscrito de Huarochirí, parece ser realizado de manera generalizada actualmente con la forma -ta, sin alomorfos (Cerrón-Palomino 2008).
} 
(15) Aymara (aymara) (Hardman, Vásquez y Yapita 2001)

a. Jani-w chur-k-i-ti.

NEG-TOP dar-INCOMPL-3SG-NEG

'No (se lo) dio.'

b. Jani-w NEG-EVID kawkha-n-s

dónde-LOC-ADIT chur-k-i-ti. dar-INCOMPL-3SGNEG

'No (se lo) dio en ninguna parte.'

Hay otras lenguas en las que los negativos indefinidos no ocurren simultáneamente con la predicación negativa (16a-b); si se llegara a dar esta ocurrencia simultánea, la oración sería agramatical $^{11}$ (Haspelmath 2013).

(16) Alemán (indoeuropea, germana) (Haspelmath 2013)
a. Niemand kam. nadie vino 'Nadie vino.'
b. Ich habe nichts gesehen. yo he nada visto 'No he visto nada.'

El español tiene un comportamiento mixto. Hay casos donde los indefinidos aparecen antes del verbo y operan como negación predicativa (17a), pero cuando el negativo indefinido aparece después del verbo, la negación predicativa es requerida (17b):

(17) Español (indoeuropea, romance) (Ejemplo propio)
a. Nadie lleg-ó.
b. No lleg-ó
nadie. nadie llegar-3SG.IND.PRES 'Nadie llegó.' NEG llegar-3SG.IND.PRES nadie 'No llegó nadie.'

Otras lenguas, como el nêlêmwa (18), tienen construcciones negativas existenciales, las que exhiben un negativo o verbo negador existencial como predicado principal. En este caso, el pronombre indefinido es el sujeto (Haspelmath 2013).

11 Formas como *niemand kam nicht son agramaticales. 
(18) Nêlêmwa (austronésica, oceánica) (Haspelmath 2013)

Kia agu i uya.

no_existe persona 3sG venir

'Nadie vino.' (Lit. 'no existe una persona que venga / haya venido')

La tendencia general muestra que las lenguas presentan ocurrencia simultánea entre pronombre negativo indefinido y marca de negación predicativa. Los comportamientos mixtos, la construcción negativa existencial y la aparición de negativos indefinidos que anteceden a la negación predicativa son mucho menos frecuentes (Haspelmath 2013).

\subsection{La negación no estándar}

En cuanto a la negación no estándar, el trabajo de van der Auwera y Lejeune (2013) propone cuatro tipos de negación en imperativos. El primero se observa en (19) y (20); aquí la negación de indicativo y la de imperativo utilizan la misma partícula negativa:

(19) Inglés (indo-europea, germánica) (Ejemplo propio)
a. You go.
b. You do not $\mathrm{go}^{12}$. 2 ir 2 AUX NEG ir

'Tú vas/Uds. van'. 'Tú no vas/Uds. no van'.
c. Go!
ir
¡Ve/vayan!’
d. Do not go!
AUX NEG ir
¡No vayas/vayan!’

(20) Aymara (aymara) (Cerrón-Palomino 2008)
a. Uma
apa-n-ma $a^{13}$.
agua
llevar-DIR-IMP.2SG

'Trae agua.'

\footnotetext{
12 En inglés hablado, se produce contracción del auxiliar do y la partícula negativa not, lo que origina la forma don't, lo que, sin embargo, no cambia este análisis, pues la forma negativa sigue siendo la misma en la declarativa que en la imperativa.

13 También, es posible la variante apa-ni-m (Hardman, Vásquez y Yapita 2001), formada por los mismos afijos que la forma reportada por Cerrón-Palomino.
} 
b. Jani um(a) apa-n-ma-ti.

NEG agua llevar-DIR-IMP.2SG-NEG

'No traigas agua.'

El segundo tipo se observa en el purépecha (21), quechua (22) y guaraní (23), donde notamos que la estrategia del imperativo es distinta de la de las declarativas. Tanto la imperativa afirmativa (21a) como la imperativa negativa (21b) utilizan la misma construcción, pero la marca de negación es distinta ('no en 21a y 'aši en 21b). Concordantemente, la marca negativa estándar (21c) es distinta de la imperativa negativa:

(21) Purépecha (tarascano) (Miestamo 2007: 13)
a. 'No 'xua-ø-rini 'sani.
b. 'Aši 'xua-ø-rini 'sani.
NEG traer-IMP-2 $>1$ pequeño
NEG traer-IMP-2 $>1$ pequeño
'No, ¡tráeme poco!'
‘No me traigas poco!'
c. 'No pi'ri-šin-ti.
NEG cantar-HAB-ASS.3
'Él no canta.'

De manera similar, tanto el quechua como el guaraní cuentan con estrategias distintas en las oraciones declarativas y en las imperativas. En el quechua, el negador mana, usado en las declarativas (ver ejemplos 11 y 14), es reemplazado por ama (22). Por otra parte, en guaraní la negación de las declarativas, formada típicamente por el circunfijo $n d-\ldots-i$ (ver ejemplos 5 y 13 ), es reemplazado por el adverbio antepuesto ani y el sufijo $-t(e) i$, opcional ${ }^{14}$.

(22) Quechua (quechua) (Cerrón-Palomino 2008)
a. Unu-ta
agua- ACC
apa-mu-y!
'Trae agua.'
14 Adicionalmente, el guaraní cuenta con estrategias de negación particulares para otros tipos de oraciones, por ejemplo, en tiempo futuro y en modo optativo (Krivoshein De Canese 1983). Estas estrategias son, en general, circunfijales, a excepción de la usada en el imperativo, en la cual la parte que antecede al verbo es descrita como un adverbio y no como prefijo.


b. Ama unu-ta apa-mu-y-chu!

NEG agua- ACC llevar-DIR-IMP.2SG-NEG

'No traigas agua.'

(23) Guaraní (tupí-guaraní) (Estigarribia 2017)

a. $(N d e)$ re-bo.

2SG 2SG.ACT-ir

'(Tú) vas.'

b. (Nde) ani re-bo-tei.

2SG IMP.NEG 2SG.ACT-ir-IMP.NEG

'(Tú) no vas.'

El tercer tipo lo ilustra la construcción negativa imperativa del castellano y del italiano (24d), que son distintas de las afirmativas (24c), aunque la negativa utiliza el negador estándar (24b y d).

(24) Italiano (indoeuropea, romance) (Miestamo 2007:13)
a. Canti.
b. Non canti.
cantar.IND.PRES.2sG
NEG cantar.PRES.2SG
'Cantas'
'No cantas.'
c. Canta!
cantar.IMP.2SG
'¡Canta!'
d. Non cantare!
NEG cantar.INF
¡No cantes!'

Finalmente, el cuarto tipo (25) usa prefijos personales en la imperativa negativa (25b), diferenciándose tanto de la forma verbal imperativa positiva (25a) como del marcador de negación estándar (25c):

(25) Koasati (maskogui) (Miestamo 2007:13)
a. Íp!
b. Is-p-án!
c. Cik-m-o.
comer
2SG-comer-NEG.IMP
‘ Come!'
‘No comas!'
2sG.NEG-reunir-NEG
'No te juntas/reúnes.' 
Tabla 2. Imperativas y negación estándar según van der Auwera y Lejeune (2005)

\begin{tabular}{lccc}
\hline & $\begin{array}{c}\text { Imperativa } \\
\text { afirmativa }\end{array}$ & $\begin{array}{c}\text { Imperativa } \\
\text { negativa }\end{array}$ & $\begin{array}{c}\text { Negación } \\
\text { estándar }\end{array}$ \\
\hline Tipo I (ínglés, aymara) & $\mathrm{V}_{1}$ & $\mathrm{~V}_{1} \mathrm{NEG}$ & $\mathrm{V}_{2} \mathrm{NEG}$ \\
$\begin{array}{l}\text { Tipo II (purépecha, guaraní, } \\
\text { quechua) }\end{array}$ & $\mathrm{V}_{1}$ & $\mathrm{~V}_{1} \mathrm{NEG}_{1}$ & $\mathrm{~V}_{2} \mathrm{NEG}_{2}$ \\
Tipo III (castellano, italiano) & $\mathrm{V}_{1}$ & $\mathrm{~V}_{2} \mathrm{NEG}$ & $\mathrm{V}_{3} \mathrm{NEG}$ \\
Tipo IV (koasati) & $\mathrm{V}_{1}$ & $\mathrm{~V}_{2} \mathrm{NEG}_{1}$ & $\mathrm{~V}_{3} \mathrm{NEG}_{2}$ \\
\hline
\end{tabular}

En síntesis, los cuatro tipos de negación imperativa son (a) la construcción imperativa negativa emplea las mismas estrategias en construcciones declarativas estándar; (b) la construcción imperativa negativa utiliza una estrategia de negación distinta a la negación estándar; (c) la imperativa negativa usa una construcción verbal diferente a la imperativa positiva y la estrategia de negación es la misma para declarativas y (d) la imperativa negativa tiene una construcción verbal diferente a la imperativa positiva y utiliza una estrategia de negación distinta a las declarativas. A continuación, estos tipos aparecen resumidos esquemáticamente en la tabla 2.

Otro dominio de la negación no estándar lo encontramos en las cláusulas no verbales, es decir, construcciones donde el predicado no es un verbo léxico sino otro elemento (p.e. sustantivo, adjetivo o frase locativa). Estas cláusulas frecuentemente exhiben maneras especiales de expresar la negación. En (26) se observa una construcción no copular que usa un negador especial bukan en lugar del estándar tidak.

(26) Indonesio (malayo-polinesio) (Dahl 2011:27)

Itu bukan jeruk.

esto NEG naranja

'Esto no es una naranja.'

En construcciones copulares, puede darse el caso de que la cópula sea reemplazada por una de naturaleza negativa, como en (27a-b): 
(27) Checo (indoeuropea, eslávica) (Dahl 2011: 28)
a. Jan je doma.
Juan COP.3sg en_casa
b. Jan neni
doma.
'Juan está en casa.'
Juan NEG.COP.3sG en_casa
'Juan no está en casa.'

Las construcciones negativas existenciales son otro tipo de negación no estándar. En turco, se establece una diferencia entre la forma existencial afirmativa y la negativa: var 'existe' vs. yot 'no existe' (Dahl 2011). Otra posibilidad implica que el verbo negativo se convierta en una partícula en la negación existencial (28).

(28) Tzutujil (maya) (Miestamo 2007: 14)
a. $M-i x \quad$ utz ta.
NEG-2PL.ABS bien IRR
b. Ma k'o ta jay.
'Ustedes no están bien.'
NEG EX IRR casa
'No hay ninguna casa.'

De manera similar, el kawésqar exhibe una estrategia no estándar para la predicación posesiva, la que se basa en una construcción copular con el verbo jenák 'ser, estar' y las postposiciones táwon 'con', en el caso afirmativo, y kiáwel 'sin', en la construcción negativa (29):

(29) Kawésqar (aislada) (Aguilera y Tonko 2006)

a. Če sa afčár táwon jenák.
yo top leña con estar
'Yo tengo leña' (lit. 'estoy con leña').

b. Če sa afčár kiáwel jenák.
yo top leña sin estar
'Yo no tengo leña' (lit. 'estoy sin leña').

Finalmente, el mismo elemento puede ser usado como negador en construcciones existenciales y en predicaciones ordinarias ("no llegó’ vs. 'no hay pan') (Miestamo 2007, Croft 1991). 


\section{El mapudungun}

La lengua mapuche o mapudungun tiene actualmente alrededor de 250.000 hablantes en la zona centro-sur de Chile y el centro de Argentina (Eberhard, Simons y Fenning 2020). Es posible distinguir diversas variedades dialectales, pero las diferencias entre ellas son más bien modestas, pese a ocasionales aseveraciones en sentido contrario. Esta es una de las varias razones para discrepar del Ethnologue y el Glottolog y no postular una familia lingüística "araucana"; consideramos la lengua como aislada o no clasificada. En el presente trabajo, nos concentramos en la variedad llamada mapudungun. En cuanto a sus convenciones ortográficas, existen varios "grafemarios", tanto en publicaciones académicas como literarias. Aquí usamos el llamado Alfabeto Mapuche Unificado.

En cuanto a sus características tipológicas, la morfología del mapudungun puede clasificarse como sintética-polisintética. La morfología nominal es relativamente simple, con muy poca flexión y solo limitada afijación derivacional, pero con uso extenso de patrones de composición. Por el contrario, la morfología verbal es extremadamente compleja: la afijación léxica es más bien limitada, pero hay numerosas posiciones morfemáticas en la plantilla morfológica, según Smeets (2008) más de treinta, la incorporación nominal continúa siendo bastante productiva y es común el uso de bases verbales que constan de varias raíces (Zúñiga 2017). En general, predomina, en su morfología, la sufijacion y "aglutinación" (es decir, no hay flexividad; la mayor parte de los morfemas muestran exponencia simple y se combinan de modo concatenativo para formar palabras). Respecto de la sintaxis, existen cláusulas nominales y verbales. Las últimas distinguen dos tipos de cláusulas transitivas (directas e inversas, Haude y Zúñiga 2016). Los patrones de orden de constituyentes parecen haber sido predominantemente del tipo VSO en el pasado; posiblemente bajo la influencia del intenso contacto con el español del Cono Sur, los patrones del tipo SVO son más comunes actualmente (Zúñiga 2006b). 


\subsection{La negación en las gramáticas del mapudungun}

Antes de pasar al análisis de las estrategias de negación del mapudungun, haremos una revisión histórica del tema en las gramáticas de la lengua.

En su obra de 1606 (Cap IX), Luis de Valdivia señala que existen tres "partículas" en la lengua, las cuales se interponen a los verbos para generar la negación. Estos elementos "son la, qui, no" (Valdivia [1606] 1684: 26). Explica que la primera partícula la solamente se pone en el indicativo y no en otro modo (v.gr. elun 'yo doy' vs. elulan 'yo no doy'). Valdivia advierte dos detalles interesantes, el primero relacionado con la ubicación del negador y el segundo con el hecho de que algunas personas ponen la partícula -no- en lugar de la (v.gr. elupononche 'yo no doy', eluponolu 'que no da', Valdivia [1606] 1684: 26). En cuanto a la negación imperativa, explica que para realizarla es necesario el uso de la partícula $l$ del subjuntivo, a la que le antecede la forma qui. Señala, además, que el imperativo negativo está integrado en el paradigma del modo subjuntivo. Finalmente, la partícula no es utilizada para negar subjuntivos y formas no finitas; además, indica que este elemento aparece antes de la partícula li.

En Febrés ([1765] 1846), se presentan las formas de negación en el plan de conjugación del verbo y se explicitan los tres elementos ya mencionados por Valdivia. Havestadt ([1775] 1883), también, se refiere al verbo negativo y distingue tres "partículas" que se insertan en el verbo según su modo: -la para indicativo, -qui para imperativo y -no, que funciona con subjuntivo y formas no personales. Febrés, también, advierte que para la realización del imperativo negativo se utiliza la partícula - $l$ del subjuntivo. La Grasserie (1898: 34) se refiere a la negación en el marco de la conjugación verbal, presentándola como voz activa negativa. También, distingue las tres formas de negación, identificadas con las mismas "partículas" y hace la misma observación respecto del imperativo negativo.

Augusta (1903) evidencia lo mismo que ya se ha mencionado, agregando los "adverbios" negativos (v.gr. no y $m u$ ) y los nega- 
tivos indefinidos (v.gr. kiñe no rume 'ninguno, ni uno', inei no rume 'nadie', chem no rume 'nada'); al respecto señala que “ordinariamente exijen negación del verbo" (Augusta 1903: 87). Moesbach ([1963] 2013) sigue una línea similar a sus predecesores: presenta las formas negativas en los respectivos paradigmas indicativo, subjuntivo e imperativo. En cuanto a los adverbios de negación, resulta interesante la mención — por parte de Moesbach — de un adverbio negativo primario ( $m \ddot{u}$, me o $m u$ ), que servía para responder a una pregunta, aunque reconoce que es mucho más frecuente el uso de la forma negativa del verbo (¿allkü-ymi?, mü o allkü-la-n ‘¿escuchaste?, no o no escuché'). Agrega que la forma no del castellano ha reemplazado al elemento negativo primario.

Por su parte, Smeets (2008: 243) señala que a la negación le corresponde el décimo casillero funcional, que puede ser llenado por -la,-ki o -nu. El primero ocurre en formas de indicativo; el segundo, en formas de imperativo y obligatoriamente se combina con $-l$ del condicional. Puede ocurrir ocasionalmente la secuencia $k i$-nu-l en lugar de - $k i$ - $l$, lo que implicaría un problema, pues combina dos marcadores de negación y dos modos; sin embargo, afirma Smeets, esta combinación no es frecuente. Por otro lado, la autora sostiene que el paradigma de imperativo intransitivo no está completo, ya que no hay forma imperativa para primera persona sujeto no singular (dual y plural). Estas formas pueden ser utilizadas exhortativamente en el indicativo; sin embargo, la forma negativa de indicativo es reemplazada por $-k i-l$ para indicar negación cuando se usa con función exhortativa. Por último, el sufijo -nu ocurre en formas condicionales, en subordinadas y en construcciones nominales, la que alternativamente también puede manifestarse como -no. Además, puede usarse como negador de construcciones que contienen rume 'siempre'. En este punto, Smeets discrepa de Augusta, quien habla de indefinidos negativos (v.gr. iney no rume 'nadie'). Finalmente, menciona que el adverbio turpu 'nunca' tiende a combinarse con verbos negativos.

Salas (2006) menciona las mismas estrategias de negación ya revisadas. Sin embargo, distingue la negación en subjuntivo hipotético 
negativo, donde se incorpora el morfema $n u$ o $-n o$, como en $p i$ no-l-mi mi ruka lif nieael 'si no quieres tener tu casa limpia' (Salas 2006: 146), del subjuntivo volitivo negativo, que se realiza a través del sufijo - $k i$, como en kon-ki-l-iñ '¡no entremos!' (Salas 2006:146). Estas formas pueden reforzarse opcionalmente agregando el morfema -no -nu, lo que resulta en kon-kino(nu)-l-iñ. Por último, Salas señala que el sufijo - $k i$ puede ser opcionalmente reforzado con $-n o \sim-n u$, con ocurrencia obligatoria del sufijo - $l$ del subjuntivo (irrealis). En su síntesis, señala que la lengua niega por medios morfológicos y distingue entre las tres formas -la para indicativo, $-n u$ o -no para subjuntivo hipotético, y -ki...-l o -kino...-l tanto para el subjuntivo volitivo como para para el modo imperativo.

Harmelink (1996: 104) reconoce los mismos medios morfológicos para establecer la negación que se han señalado anteriormente. Un aspecto relevante de Harmelink (1996: 173) dice relación con la combinación de morfemas y la negación en indicativo. El autor señala que al combinar los morfemas - ke y -la, estos dan el significado de 'nunca' (küpa-ke-la-ymi 'nunca vienes', Harmelink 1996:173), por lo que recomienda pensar en el sentido de la secuencia y no en el significado separado de los morfemas. Hace el mismo comentario respecto de la combinación de las partículas -we y -la, en ese orden, que daría el sentido de 'ya no' (küpa-wela-ymi ‘ya no vienes', Harmelink 1996: 173). En ambos casos, se observa un conjunto natural de morfemas que dan un sentido que vale tomar de modo combinado.

En cuanto al uso del adverbio turpu, este autor sostiene que es un recurso que sirve para dar mayor énfasis a la negación (amu-ke-la-n 'nunca voy' vs. turpu amu-ke-la-n 'nunca jamás voy'; Harmenlink 1996: 184). En este mismo dominio adverbial, agrega dos elementos que solo funcionan en cláusulas negativas, müte y newe, cuyo significado se interpreta como 'no muy/mucho' (müte küme-la-y 'no es muy bueno'; newe wütre-nge-la-y 'no hace mucho frío', Harmenlink 1996: 184).

En cuanto a los pronombres indefinidos, Harmelink (1996: 57) señala que la manera de decir 'nada', 'ninguno' y 'nadie' es por 
medio de las formas chem no rume, kiñe no rume e iney no rume respectivamente y presenta los siguientes ejemplos: nge-la-y chem no rume challa mew 'no hay nada en la olla', kiñe no rume niela-n 'no tengo ninguno' e iney no rume küpa-la-y 'no vino nadie'. Agrega que la función de rume es reforzar el sentido negativo de la oración.

Para Adelaar y Muysken (2004), la negación se indica por medio de la morfología. Distinguen los mismos tres marcadores y el tipo de distribución ya mencionado por Smeets (2008). Un elemento que se debe destacar es la idea de que en los predicados nominales nu es un elemento libre o un clítico antes que un sufijo (fey domo=nu 'él no es mujer').

Zúñiga (2006: 110) introduce el tema a partir de la noción de polaridad; muestra las diferencias existentes entre el español y el mapudungun, afirmando que el último forma la negación por medio de un sufijo verbal, el cual es diferente según el modo -la en indicativo, $-n o /-n u$ tanto para el subjuntivo como para las formas no finitas y $-k i$ para el modo imperativo. Finalmente, agrega, pueden hallarse construcciones que utilizan tanto - $k i$ como -no.

Por su parte, Catrileo (2010: 126) señala la existencia de los indefinidos negativos, los que escribe sin separación de palabras: ineynurume 'nadie' y chemnurume 'nada' (i.e. iney nu rume, y chem nu rume, respectivamente). Hace referencia a la negación en cláusulas nominales Kuannu 'no es Juan' y adjetivas wedwednu 'no es un necio'. Además, agrega que la negación con $n u$ también se aplica a adverbios (matunu 'no es urgente'), pronombres (inchenu 'no soy yo'), postposiciones (locativas) (ruka mewnu 'no es en la casa') y formas condicionales (nie-nu-le 'si él o ella no tiene'). En cuanto al imperativo, esta autora señala que existe la marca - kil para la forma negativa y agrega que puede darse una doble negación -kinul, la que indicaría mayor énfasis. Por último, señala la existencia de la doble negación en la lengua, cuyo propósito es la expresión cabal del sentido negativo, por lo que aplica la negación no solo al lexema verbal sino también a los constituyentes de la construcción v.gr. 
turpu feyentu-ke-la-y 'nunca obedece' o chumkawnurume feypila-ya-n 'no lo diré bajo ninguna circunstancia' (Catrileo 2010: 129).

Finalmente, Loncón (2011: 90-91) presenta la negación bajo el título de oraciones negativas. Señala que la negación con el afijo -la es la más frecuente en la lengua, y muestra la negación con el afijo $-n u /-n o$ en el modo condicional y la negación en imperativo con $-k i \ldots-l$.

\section{La investigación}

Los objetivos de esta investigación son (1) dar cuenta de las estrategias de negación de la lengua mapuche y (2) presentar una clasificación de las construcciones negativas del mapudungun hablado en el territorio de la Araucanía (Chile) de acuerdo con los planteamientos tipológicos contemporáneos. Para lograr estos propósitos, se analizaron los casos presentados en la literatura y se aplicó una lista de construcciones negativas basadas en el cuestionario de la negación de Miestamo (2016).

\subsection{Metodología}

La técnica escogida para recoger los datos fue la elicitación de construcciones negativas a partir de la aplicación de un cuestionario de construcciones negativas que cuenta con un total de 50 estímulos (se complementó con datos tomados de las publicaciones disponibles sobre la lengua). Este instrumento se orienta a la caracterización de las estrategias de negación estándar y no estándar en diversos tipos de construcciones: finitas, no finitas y no verbales. En el marco de las construcciones finitas, se prestó atención a las formas de negación predicativas y estativas, concentrándose en las indicativas, subjuntivas e imperativas, del primer grupo, y las atributivas, locativas, inclusivas propias, existencial y posesivas, del segundo. Finalmente, se observa el comportamiento de los indefinidos negativos. 
El instrumento se aplicó en 6 hablantes bilingües ${ }^{15}$ mapucheespañol, quienes se adscriben a los territorios de Loncoche Plom, Isla Huapi y Cruzaco. El primero se encuentra a 20 kilómetros de Temuco en dirección suroeste. La zona de Isla Huapi está a una distancia de 100 kilómetros de Temuco, hacia la costa de la región de la Araucanía. El territorio pertenece a la comuna de Saavedra. Por último, la zona de Cruzaco se ubica a 144 kilómetros de Temuco, hacia la cordillera de los Andes, la zona es parte de la comuna de Lonquimay. Los hablantes de Loncoche Plom e Isla Huapi se adscriben a la variedad dialectal etiquetada como mapuche central, mientras que los participantes de Cruzaco tienen la variedad pehuenche, hablada en la zona cordillerana del territorio chileno-argentino.

El análisis se orienta a determinar cuáles son los esquemas de negación prototípicos vigentes que enuncian los hablantes.

\section{Descripción delas construcciones negativas del mapudungun}

Esta descripción parte de la siguiente pregunta: ¿Cómo se codifica la negación en la lengua mapuche? ¿Cuáles son sus estrategias?

\subsection{La negación estándar}

En cuanto a la negación estándar, como ya bien se ha señalado en la literatura revisada, el morfema de negación estándar del mapudungun es el sufijo -la, tal como se observa en (30) y (31):

(30) mapudungun (aislada)

a. Feyti trewa wangkü-y.

DET perro ladrar-IND[3sG]

'Ese perro ladró.'

b. Feyti trewa wangkü-la-y.

DET perro ladrar-NEG-IND[3SG] 'Ese perro no ladró.' (H1IH $\left.{ }^{16}\right)$

\footnotetext{
15 Agradecemos a los colaboradores por haber participado en este trabajo. Sin su conocimiento y su generosidad, este estudio no podría haberse realizado.

16 La codificación de los colaboradores se hizo del siguiente modo: Colaboradores 1 y 2 de Isla Huapi (IH), Loncoche Plom (LP), Cruzaco (C): C1IH, C2IH, C1LP, C2LP, C1C, C2C.
} 
(31)
a. Nie-fu-y küla fotüm. tener-APRF-IND[3SG] tres hijo '(El hombre) tenia tres hijos.'
b. Feychinie-la-fu-y tralka. DET tener-NEG-APRF-IND[3sG] escopeta
'Aquel no tenía escopeta.' Kon-ke-y küllon.
entrar-HAB-IND[3SG] maqui
'Entra (=se incluye el) maqui.'
d. Kon-ke-la-y ñi ngülam. entrar-NEG-HAB-IND[3SG]POS.3 consejo 'No entra (=no es considerado) su consejo.' (Coña 2000)

Se observa que la negación estándar es simétrica, pues la construcción negativa no difiere estructuralmente de la afirmativa, tanto en la forma básica del verbo, que no considera morfemas tempoaspectuales, y que tiene una interpretación de no futuro (30), como en verbos con distintos morfemas tempo-aspectuales (31).

\subsection{La negación no estándar}

Como se evidenció en la revisión bibliográfica, el mapudungun cuenta con alomorfos para expresar la negación en los distintos modos, en las formas no finitas y en las cláusulas no verbales.

5.2.1. Negación en modos subjuntivo e imperativo

Según nuestra revisión bibliográfica, el mapudungun tiene dos alomorfos para marcar la negación en los modos subjuntivo e imperativo. En (32b), se observa la negación en el modo subjuntivo.
a. Tripa-l-mi wakon kutran-a-ymi. salir-SBJv-2sG casa enfermar-FUT-IND[2SG]
'Si sales de la casa, te enfermarás.' (C2IH)
b. Tripa-nu-l-imi wakon fey kumelka-le-a-ymi. salir-NEG-SBJV-2SG casa entonces bien-EST-FUT-IND[2SG] 'Si no sales de la casa, entonces vas a estar bien.' (C2IH)

Se observa que el morfema de negación en cláusulas de subjuntivo es -nu, que puede aparecer también como -no. 
En cuanto al modo imperativo, la negación se marca por medio del sufijo -ki (33b) y (34c), o -ki-nu (34b y d):
a. iTripa-nge!
salir-IMP $[2 \mathrm{SG}$
b. ¡Tripa-ki-l-nge!
¡Sal! (C1C)
salir-NEG-SBJV-IMP[2SG]
¡No salgas! (C1C)

(34)
a. iLa-pe!
morir-IMP.[3]
b. ¡LLa-ki-nu-l-pe pu machi!
¡Que muera! morir-NEG-NEG-SBJV-IMP[3] PL machi ¡Que no muera, oh machis!
hacer.así-NEG.SBJV.IMP.[2sG]
¡No hagas eso!
c. iFem-ki-l-mi!
d. ¡Welu fem-ki-nu-l-mün! pero hacer.así-NEG-NEG-SBJV-IMP.[2SG] ¡Pero no hagan eso! (Coña 2000)

Este paradigma es el que presenta mayor grado de variación en la lengua. Al respecto, Zúñiga (2018) señala que el paradigma de imperativo negativo pasa por un proceso de variación que, al parecer, ya no establece la división etimológica entre $-k i$ y $-n u$, fenómeno que se evidencia por el uso de formas con doble marca -ki-nu e, incluso, por el uso de nu en el imperativo. Agrega que el alomorfo -nu se encuentra en un proceso de expansión hacia el paradigma de imperativo, posiblemente reforzado por el modelo del español, cuyo paradigma de imperativo ocupa la forma de segunda persona.

\subsubsection{Negación en formas no finitas}

El alomorfo $-n u \sim-n o$ es utilizado para negar las formas no finitas, como se aprecia en (35):
a. Amu-a-lu Kuan. ir-FUT-NFIN Juan 'Juan va a ir.'
b. Amu-no-a-lu Kuan. ir-NEG-FUT-NFIN Juan 'Juan no va a ir.' $(\mathrm{C} 1 \mathrm{IH})^{17}$

\footnotetext{
17 La forma no finita con sufijo - lu puede utilizarse en lugar de la forma finita (en este ejemplo: amu-la-ya-y) para referirse al futuro. Las razones de dicha alternancia no han sido del todo esclarecidas.
} 
c. Feyti kawellu tami ngilla-el rume newe-nge-y.

DET caballo POS.2SG comprar-NFIN muy fuerte-ser-IND[3sG] 'El caballo que compraste es muy fuerte.' ${ }^{\text {'18 }}$

d. Feyti kawellu tami ngilla-nu-el rume newe-nge-y. DET caballo POS2SG comprar-NEG-NFIN muy fuerte-ser-IND[3sG] 'El caballo que no compraste es muy fuerte.'

\subsection{Negación en construcciones estativas}

Antes de pasar a las construcciones estativas, considérese la existencia de dos verbos copulativos en mapudungun: ngen 'ser, existir' que es un predicado de estado permanente y mülen 'estar', de estado transitorio.

\subsubsection{Inclusión propia/adscriptivas}

Se expresa a través de la verbalización con -nge 'ser':
a. Kimeltuchefe-nge-y. profesor-ser-IND[3sG] 'Es un profesor.'
b. Kimeltuchefe-nge-la-y. profesor-ser-NEG-IND[3SG] 'No es un profesor.' (C2HP)

Se observa que la negación se expresa a través del negador estándar.

\subsubsection{Atribución}

En las cláusulas atributivas, la negación usa la marca estándar -la. Las construcciones se caracterizan por usar el sufijo -nge $(<n g e n)$ 'ser' (37a) y -le (< mülen) 'estar' (37b).
a. Motri-nge-y Fernando. gordo-ser-IND[3sG] Fernando 'Fernando es gordo.'
c. Motri-le-y
Fernando. gordo-EST-IND[3SG] Fernando 'Fernando está gordo.'
b. Motri-nge-la-y
Fernando. gordo-ser-NEG-IND[3sG] Fernando 'Fernando no es gordo.'
d. Motri-le-la-y
Fernando. gordo-EST-NEG-IND[3SG] Fernando 'Fernando no está gordo.'

18 Conocimiento propio. 
5.3.3. Construcciones estativas locativas

En las estativas locativas, también, se utiliza la marca de negación estándar la, como se observa en (38): ${ }^{19}$

a. Feyti ruka müle-y wingkul mu.

DET casa estar-IND[3sG] cerro POSP

'La casa está en el cerro.'

b. Feyti ruka müle-la-y mawida mu.

DET casa estar-NEG-IND[3SG] monte POSP

'La casa no está en el monte.' (C1C)

5.3.4. Construcciones existenciales

Para expresar el sentido de existencia, el mapudungun utiliza los verbos ngen 'ser' y mülen 'estar', los cuales dan cuenta de una oposición basada en la polaridad. Al comparar los ejemplos en (39), puede observarse el uso ya sea positivo o negativo de mülen pero solo negativo de ngen (aunque el ámbito de función de este último elemento es más amplio), conformando una dupla asimétrica:
a. Müle-y pu sañue korral mu. existir-IND[3sG] PL cerdos corral POSP 'Hay cerdos en el corral.'
b. Müle-la-y pu sañue korral mu. existir-NEG-IND[3SG] PL cerdos corral POSP
'No hay cerdos en el corral.'
c. Nge-la-y sañue korral mu. 'No hay cerdos en el corral.' (C1IH) existir-NEG-IND[3SG] cerdos corral POSP

Sin embargo, en ciertos contextos tempo-aspectuales la construcción con el verbo ngen acepta, también, expresiones afirmativas (40), convirtiéndose en simétrica en dichos contextos reducidos.

19 Las glosas y las traducciones no son erróneas, tanto wingkul 'cerro' como mawida 'montaña' pueden utilizarse para referirse a cerros. 
Estos son especialmente contextos marcados con el morfema de antiperfecto $-f u$, antiperfecto más futuro $-a-f u$, o epistémico más antiperfecto - $p e-f u$ :

a. Doy mari nge-pe-fu-y-ngün.

más diez existir-EPI-APRF-NEG-IND-[3PL]

'Habría más de diez."

b. Küme-ke ad-nge-fu-yngün wesha-ke ad ka nge-fu-yngün. bueno-PL rasgo-ser-APRF-IND[3PL] malo-PL rasgo también existir-APRF-IND[3PL]

'Tenían buenas características y también tenían malas características (costumbres)'. (Coña 2000)

\subsubsection{Construcciones posesivas}

El mapudungun presenta dos maneras de expresar la posesión predicativa (Olate et al. 2018), la primera a través del verbo nien 'tener ${ }_{1}$, que expresa posesión permanente, y otra con la sufijación de $n g e$ 'tener ${ }_{2}$ / estar con', que expresa posesión temporal. En español estándar, ambos tipos de posesión se expresan por medio del verbo 'tener'; coloquialmente se puede decir 'estar con' en el segundo caso. La negación de este tipo de construcciones utiliza la marca estándar -la. En (41a-b) observamos la expresión de la posesión permanente y su forma negada, mientras que en (41 c-d) observamos la posesión transitoria:
a. Nie-y
ruka María. b. Nie-la-y
ruka María. tener-IND[3sG] casa María 'María tiene casa.' tener-NEG-IND[3SG] casa María 'María no tiene casa.'
c. Kure-nge-y esposa-ser-IND[3sG] Juan
Kuan. d. Kure-nge-la-y
Kuan. 'Juan está con esposa.' esposa-ser-NEG-IND[3sG] Juan 'Juan no está con esposa.'

\subsubsection{Cláusulas nominales}

Estas construcciones se utilizan para expresar relaciones ecuativas. La negación en estas se realiza a través de la partícula negativa $n u$ 
o no. En (42b) y (42d), podemos observar cómo no aparece al final del elemento que está en foco, en este caso, del elemento que está siendo negado.

a. Fey inche tañi ñuke. b. Fey iñche tañi ñuke no. DEM 1SG POS[1sG] mamá DEM 1SG POS[1sG] mamá NEG 'Ella es mi mamá.' 'Ella no es mi mamá.'

c. Feychi wentru tañi peñi. d. Feychiwentru tañi peñi no. DEM hombre POS[1SG] hermano DEM hombrepos[1sG] hermano NEG 'Él es mi hermano.' 'Él no es mi hermano.'

e. Maria no tañi ñuke. f. Mapuche no iñche.

M. NEG POS[1sG] mamá mapuche NEG yo

'María no es mi mamá.' 'Yo no soy mapuche.'

En resumen, la expresión de la negación en las construcciones estativas del mapudungun se sintetiza en la siguiente tabla:

Tabla 3. Síntesis construcciones de predicación estativa (verbales y no verbales), indicativo

\begin{tabular}{|c|c|c|c|c|}
\hline & \multicolumn{2}{|c|}{ Estado permanente } & \multicolumn{2}{|c|}{ Estado transitorio } \\
\hline & Afirmativa & Negativa & Afirmativa & Negativa \\
\hline Locativa & & & $V_{-}$ & $\sqrt{ }-l a-$ \\
\hline Atributiva & $\sqrt{ }(-n g e)-$ & $\sqrt{ }(-n g e)-l a-$ & $\sqrt{ }-l e-$ & $\sqrt{ }-l e-l a-$ \\
\hline Existencial & nge- & nge-la- & $\begin{array}{l}\text { müle- } \\
\text { nge-la-20 }\end{array}$ & $\begin{array}{l}\text { müle-la } \\
\text { nge-la- }\end{array}$ \\
\hline Posesivas & nie- & nie-la- & \multirow{2}{*}{ NP-nge- } & \multirow{2}{*}{ NP-nge-la- } \\
\hline Inclución nroni & NP-nge- & NP-nge-la- & & \\
\hline Ecuativas & NP NP & NP NP $\boldsymbol{n} \boldsymbol{u}$ & & \\
\hline
\end{tabular}

20 En contextos tempo-aspectuales específicos. 


\subsection{Indefinidos negativos}

En cuanto a los indefinidos negativos, de acuerdo con la tipología de Haspelmath (2013), la tendencia general muestra que las lenguas presentan ocurrencia simultánea de negativo indefinido y la marca de negación predicativa. En mapudungun, la ocurrencia simultánea puede darse o no. No es este el espacio que resolverá esta cuestión. El fenómeno debe explorarse con mayor profundidad, ya que el componente pragmático puede condicionarlo.

En la discusión bibliográfica, sin embargo, nos encontramos con tres autores que plantean que en mapudungun hay ocurrencia simultánea. Para Harmelink, rume tiene la función de reforzar el sentido negativo de la oración:

a. Nge-la-y chem no rume challa mew. existir-NEG-IND[3SG] algo NEG siquiera olla POSP 'No hay nada en la olla.'

b. Kiñe no rume nie-la-n. uno NEG siquiera tener-NEG-IND[1sG]

'No tengo ninguno.'

c. Iney no rume küpa-la-y. quien NEG siquiera venir-NEG-IND[3SG]

'No vino nadie.' (Harmelink 1996: 57)

Por su parte, Augusta (1903: 87) señala que los negativos indefinidos comúnmente exigen la negación del verbo. Finalmente, Catrileo (2010: 129) señala que la doble negación tiene la función de lograr un sentido negativo cabal para el receptor.

En nuestro corpus, se presentó el uso concurrente de negación morfológica verbal e idefinido negativo, mostrado a continuación (44), concordante con lo planteado tanto por Harmelink (2006) como por Catrileo (2010):

a. Ini no rume küpa-la-y pe-a-lu ta füta che.

Quien NEG siquiera venir-NEG-IND[3SG] ver-FUT-NFIN ENF mayor gente 'Nadie vino a ver al abuelito.' (C2IH) 


\section{Conclusiones}

En este trabajo hemos presentado las estrategias y formas de la negación en la lengua mapuche. En la tabla 4, se observan las formas negativas del mapudungun:

Tabla 4. Formas negativas del mapudungun

\begin{tabular}{|c|c|c|c|c|}
\hline \multicolumn{4}{|c|}{ Verbal } & No verbal \\
\hline \multicolumn{3}{|c|}{ Finito } & No finito & \\
\hline Imperativo & Indicativo & Subjuntivo & & \\
\hline$-\mathrm{ki}$ & $-\mathrm{la}$ & $-\mathrm{nu} \sim-$ no & $\mathrm{nu} \sim$ no \\
\hline
\end{tabular}

Por otro lado, hemos querido presentar una clasificación de las construcciones negativas de la lengua de acuerdo con la tipología contemporánea. $\mathrm{Al}$ respecto, podemos señalar:

1. A partir del criterio de la simetría o asimetría del paradigma de negación, la lengua mapuche tiene un paradigma de negación predominantemente simétrico: casi no se advierten asimetrías funcionales en los paradigmas indicativo, subjuntivo e imperativo, a excepción de la alternancia entre el uso del verbo mülen y ngen, y del uso de ngen preferentemente en oraciones negativas y en contextos afirmativos acotados por marcadores tempo-aspectuales que indican una falta de compromiso del hablante con la afirmación de la existencia del referente en el momento presente.

2. La negación en mapudungun es de carácter mixto, aunque principalmente morfológica por medio de los sufijos $-l a,-k i$, $-n u \sim-n o$. La partícula $n u \sim$ no opera en cláusulas nominales.

3. Desde el punto de vista de la negación estándar y no estándar, la lengua presenta negación estándar a través de la forma -la para indicativo.

4. La lengua mapuche presenta también negación no estándar, pues cuenta con alomorfos para imperativo $-k i$ y para subjuntivo $-n u \sim-n o$. En este dominio se observa que el sufijo 
$-n u \sim-n o$ parece estar desplazando al imperativo ki, cuestión que se evidencia por la variación que presenta el paradigma.

5. El carácter morfológico de la expresión de la negación en mapudungun, y su distinción entre negación estándar y no estándar, la asemejan más a la negación en guaraní (que, sin embargo, difiere por realizarse por medio de circunfijos) que a la negación en kawésqar, la que utiliza una partícula libre postverbal, y a aquella en lenguas andinas centrales como el quechua y el aymara, las que utilizan doble negación por medio del uso concurrente de una partícula que precede al verbo y un sufijo verbal.

6. En cuanto a los indefinidos negativos, la lengua presenta un funcionamiento mixto, con ocurrencia simultánea de negadores y, únicamente, por negación estándar. El uso de una u otra forma probablemente esté condicionado por factores pragmáticos.

7. La negación en cláusulas estativas verbales se realiza por medio del marcador estándar la.

Para finalizar, con este trabajo enriquecemos nuestro conocimiento de las lenguas surandinas, en tanto nos permitimos situar al mapudungun en el contexto de la tipología de la negación. Los datos presentados pueden ser útiles para el establecimiento de relaciones lingüísticas areales y, sin duda, confirman la importancia y utilidad del marco tipológico para la descripción, análisis y comprensión del funcionamiento de las lenguas naturales.

\section{Referencias bibliográficas}

Adelaar, Willem y Pieter MuYsken

2004 The Languages of the Andes. Cambridge: Cambridge University Press.

Aguilera, Óscar y José Tonko

2006

Manual para la Enseñanza de la Lengua Kawésqar. Nivel Básico 1b. Punta Arenas: Fundación para el Desarrollo XII Región de Magallanes [FIDE XII]/Conadi. 
Augusta, Félix

1903 Gramática Araucana. Valdivia: Imprenta Central J. Lampert.

Catrileo, María

2010 La lengua mapuche en el siglo XXI. Valdivia: Universidad Austral de Chile.

Cerrón-Palomino, Rodolfo

2008 Quechumara. Estructuras paralelas del quechua y del aimara. La Paz: Universidad Mayor de San Simón/ProEIBAndes.

CoÑa, Pascual

2000 Lonco Pascual Coña ñi tukulpazugun. Testimonio de un cacique mapuche. Séptima edición, Santiago: Pehuén.

Croft, William

1991 “The evolution of negation”. Linguistics 27, 1-27.

DAHL, Östen

2011 “Typology of negation”. En The Expression of Negation. Ed., Horn, Laurence. Berlín: Mouton de Gruyter.

DRYER, Matthew S.

2013a "Negative morphemes". En The World Atlas of Language Structures Online. Eds., Matthew S. Dryer y Martin Haspelmath. Leipzig: Max Planck Institute for Evolutionary Anthropology. Consultado: 6 de febrero del 2019. <http:// wals.info/chapter/112 $\geq$

DRYER, Matthew S.

2013b "Order of negative morpheme and verb". En The World Atlas of Language Structures Online. Eds., Matthew S. Dryer y Martin Haspelmath. Leipzig: Max Planck Institute for Evolutionary Anthropology. Consultado: 6 de febrero del 2019. $<$ http://wals.info/chapter/143 $\geq$

DRYer, Matthew S.

2013c "Position of negative morpheme with respect to subject, object, and verb". En The World Atlas of Language Structures Online. Eds., Matthew S. Dryer y Martin Haspelmath. Leipzig: Max Planck Institute for Evolutionary Anthropology. Consultado: 6 de febrero del 2019. <http://wals.info/chapter/144 $\geq$ 
Eberhard, David; Gary Simons y Charles Fenning (eds.)

2020 Ethnologue: Languages of the world, twenty-third edition. Dallas, Texas: SIL International. Consultado 20 de junio de 2020.:http://www.ethnologue.com

ESTIGARRIBIA, Bruno

2017 "A grammar sketch of Paraguayan Guarani”. En Guarani Linguistics in the 21st Century. Eds., Bruno Estigarribia, y Justin Pinta. pp. 7-85. Leiden: Brill.

FEBRés, Andrés

[1765] 1846 Gramática de la lengua chilena. Santiago de Chile: Imprenta de los Tribunales.

GRONDIN, Marcelo

1999 Método de aymara. La Paz/Cochabamba: Los amigos del libro.

Hardman, Martha, Juana VÁsquez y Juan de Dios YaptTa

2001 Aymara. Compendio de estructura fonológica y gramatical. La Paz: Instituto de Lengua y Cultura Aymara.

HaRmelinK, Bryan

1996 Manual de aprendizaje del idioma mapuche. Aspectos morfológicos y sintácticos. Temuco: Ediciones Universidad de La Frontera.

Haspelmath, Martin

2013 "Negative indefinite pronouns and predicate negation". En The World Atlas of Language Structures Online. Eds., Matthew S. Dryer y Martin Haspelmath. Leipzig: Max Planck Institute for Evolutionary Anthropology. Consultado: 6 de febrero de 2019. <http://wals.info/chapter/115 $\geq$

Haude, Katharina y Fernando ZúÑIgA

2016 "Inverse systems and symmetrical voice: On languages with two transitive constructions”. Linguistics. 54. 3, 443-481.

HAVESTADT, Bernardo

[1775] 1883 Chilidúǵn sive Tractatus linguae chilensis. Leipzig, Germany: B. G. Teubner.

KIEviet, Paulus

2017 A grammar of Rapa Nui (Studies in Diversity Linguistics 12). Berlin: Language Science Press. 
Krivoshein de CANese, Natalia

1983 Gramática de la lengua guaraní. Asunción: Nemity.

La Grasserie, Raoul de

1898 Langue Auca (ou langue indigène du Chili). Grammaire, dictionnaire, textes traduits et analyses. París: J. Maisonneuve.

LONCÓN, Elisa

2011 Morfología y aspectos del mapudungun. Ciudad de México: Universidad Autónoma Metropolitana.

Miestamo, Matti

2005 Standard Negation: The Negation of Declarative Verbal Main Clauses in a Typological Perspective. Berlin: Mouton de Gruyter.

Miestamo, Matti

2007 "Negation: an overview of typological research". Language and Linguistic Compass. 1. 5, 552-570.

Miestamo, Matti

2013a "Symmetric and asymmetric standard negation". En The World Atlas of Language Structures Online. Eds., Matthew S. Dryer y Martin Haspelmath. Leipzig: Max Planck Institute for Evolutionary Anthropology. Consultado: 6 de febrero de 2019. <http://wals.info/chapter/113>

Miestamo, Matti

2013b "Subtypes of asymmetric standard negation". En The World Atlas of Language Structures Online. Eds., Matthew S. Dryer y Martin Haspelmath. Leipzig: Max Planck Institute for Evolutionary Anthropology. Consultado: 6 de febrero de 2019. $<$ http://wals.info/chapter/114>

Miestamo, Matti

2016 Questionnaire for describing the negation system of a language. Consultado: 16 de noviembre de 2018. <http://tulquest.humanum.fr/fr/node/134>

Miestamo, Matti

2017 "Negation”. En The Cambridge Handbook of Typology. Ed., Alexandra Aikhenvald. Cambridge: Cambridge University Press. <https://doi.org/10.1111/j.1749-818X.2007.00026.x> 
Moesbach, Ernesto

[1963] 2013 Idioma mapuche. Santiago de Chile, Chile: RIL.

Olate, Aldo et al.

2018 "Construcciones posesivas del mapuzugun. Una aproximación tipológica”. Revista de Lingüística Teórica y Aplicada. 56. 2, 35-63. <https://doi.org/10.4067/S0718-48832018000200035>

Payne, John

1985 "Negation. Language typology and syntactic description". En Clause Structure . Ed., Timothy Shopen. Volumen. 1. Cambridge: CUP.

Salas, Adalberto

2006 El mapuche o araucano. Santiago de Chile: Centro Estudios Públicos.

SMEETS, Ineke

2008 A Grammar of Mapuche. Berlin, Germany: Mouton de Gruyter.

TAYLOR, Gerald

2014 Método de aprendizaje de la lengua general. Para leer el manuscrito quechua de Huarochirí. Lima: Instituto Francés de Estudios Andinos.

VALDIVIA, Luis

[1606] 1684 Arte y gramática general de la lengua que corre en todo el reyno de Chile, con un vocabulario, y confesionario. Sevilla: Thomas López de Haro.

Van der Auwera, Johan y Ludo Lejeune (con Valentin Goussev).

2013 "The prohibitive". En The World Atlas of Language Structures Online. Eds., Matthew S. Dryer y Martin Haspelmath. Leipzig: Max Planck Institute for Evolutionary Anthropology. Consultado: 14 de febrero de 2019. <http://wals.info/ chapter/71>

ZúNiga, Fernando

2006a Mapudungun. El habla mapuche. Santiago de Chile: Centro de Estudios Públicos.

ZúNIIGA, Fernando

2006b Deixis and alignment: Inverse systems in indigenous languages of the Americas. Amsterdam: John Benjamins. 
ZúNIIGA, Fernando

2017 "Mapudungun". En The Oxford handbook of polysynthesis. Eds., Michael Fortescue, Marianne Mithun y Nicholas Evans. Oxford: Oxford University Press. 696-712.

ZúÑIGA, Fernando

2018 "Negation in Mapudungun”. Charla dictada en la conferencia Syntax of the World's Languages VIII, París (Francia), 3 de septiembre de 2018.

Recepción: 04/09/2019

Aceptación: 21/08/2020 